PROCEEDINGS OF THE

AMERICAN MATHEMATICAL SOCIETY

Volume 128, Number 4, Pages 975-980

S 0002-9939(99)05137-0

Article electronically published on August 5, 1999

\title{
CONORMAL VARIETIES AND CHARACTERISTIC VARIETIES
}

\author{
S. C. COUTinho, M. P. HOLlAnd, AND D. LEVCOVitz
}

(Communicated by Ken Goodearl)

\begin{abstract}
We show that the conormal variety of a quasihomogeneous hypersurface in $\mathbb{C}^{n}$, for $n \geq 4$, whose link is a $\mathbb{Q}$-homology sphere is not the characteristic variety of any $\mathcal{D}$-module.
\end{abstract}

\section{INTRODUCTION}

Let $X$ be a complex analytic manifold and $\mathcal{D}_{X}$ its sheaf of differential operators. The most important geometric invariant of a $\mathcal{D}_{X}$-module is its characteristic variety. This is a conical subvariety of the cotangent bundle $T^{\star} X$. It is well-known that the cotangent bundle is a symplectic manifold and that the characteristic variety of a $\mathcal{D}_{X}$-module is involutive with respect to the symplectic structure. Thus, there are two conditions that a subvariety of $\mathrm{T}^{\star} X$ must satisfy in order to be a characteristic variety: it must be conical and involutive.

Conormal varieties are involutive and conical. In fact they are exactly the lagrangian conical subvarieties of $\mathrm{T}^{\star} X$ (see, for example, Ge Proposition 3.1, p. $29]$ ). Furthermore, an irreducible lagrangian variety is always a component of the characteristic variety of some $\mathcal{D}_{X}$-module (see [CL]).

As pointed out by Malgrange in [Ma2, p. 9], no necessary and sufficient conditions are known for a variety to be a characteristic variety. However, an unpublished result of Kashiwara states that the conormal variety of a quadratic cone in $\mathbb{C}^{n}$, for $n \geq 5$ odd, is not a characteristic variety (see [CL for a proof). In this paper we extend Kashiwara's result with the following theorem.

1.1 Theorem. Let $n \geq 4$ and let $f \in \mathbb{C}\left[x_{1}, \ldots, x_{n}\right]$ be a quasihomogeneous polynomial. If the hypersurface $\mathcal{Z}(f)$ has only an isolated singularity at the origin and its link is a $\mathbb{Q}$-homology sphere, then its conormal variety is not the characteristic variety of any $\mathcal{D}_{\mathbb{C}^{n} \text {-module. }}$

A simple specific example of a polynomial that satisfies these hypotheses is $f=$ $x_{1}^{a_{1}}+\cdots+x_{n}^{a_{n}}$ with $n \geq 4$, and $a_{1}, \ldots, a_{n}$ pairwise coprime positive integers. See $\S 4$ for more details.

We would like to thank the referee for a remark that improved Proposition 2.1.

Received by the editors August 15, 1996 and, in revised form, August 7, 1997 and June 2, 1998.

1991 Mathematics Subject Classification. Primary 16S32; Secondary 13N10, 32C38.

The first author was partially supported by CNPq (Brazil) during the preparation of this paper.

(C)2000 American Mathematical Society 


\section{Preliminaries}

Let $f \in \mathbb{C}\left[x_{1}, \ldots, x_{n}\right]$ be a polynomial. We say that $f$ is non-degenerate if $\mathcal{Z}(f)$ is singular but has only an isolated singularity at the origin. We call $f$ quasihomogeneous if there exist positive integers $w_{1}, \ldots, w_{n}$ such that $f$ is homogeneous when we set $\operatorname{deg} x_{i}=w_{i}$. Equivalently $r f=\sum_{i=1}^{n} w_{i} x_{i} \partial f / \partial x_{i}$, for some positive integer $r$, the weighted degree of $f$.

Notation. Throughout the rest of the paper let $n \geq 4$ and let $f \in \mathbb{C}\left[x_{1}, \ldots, x_{n}\right]$ be a non-degenerate quasihomogeneous polynomial of weighted degree $r$ with respect to the weights $w_{1}, \ldots, w_{n}$. We will denote the corresponding Euler vector field by $E=\sum_{i=1}^{n} w_{i} x_{i} \partial / \partial x_{i}$.

Let $V$ be the space $\mathbb{C}^{n}$ with its analytic topology and $C$ the hypersurface $\mathcal{Z}(f)$. Note that $C^{\prime}=C \backslash\{0\}$ is a smooth divisor on $V^{\prime}=V \backslash\{0\}$. The conormal variety of $C$, denoted $\mathrm{T}_{C}^{\star} V$, is the closure in $\mathrm{T}^{\star} V$ of the conormal bundle of $C^{\prime}$ in $\mathrm{T}^{\star} V^{\prime}$. It is a well-known fact that $\mathrm{T}_{C}^{\star} V$ is a lagrangian conical variety.

Note that since $f$ is quasihomogeneous and non-degenerate, it is easy to calculate the fibres of $\mathrm{T}_{C}^{\star} V$ everywhere, except at the origin. However, since $\mathrm{T}_{C}^{\star} V$ is irreducible and lagrangian, it follows that it cannot contain the whole fibre at the origin, denoted by $\mathrm{T}_{0}^{\star} V$. This will eventually lead to the contradiction that will allow us to prove the theorem of $\S 1$.

Consider $\mathcal{O}_{V}\left[f^{-1}\right]$; it is naturally a coherent $\mathcal{D}_{V}$-module. We will show that the characteristic variety of this module contains the fibre $\mathrm{T}_{0}^{\star} V$. To do this we will make use of a description of the characteristic variety due to Kashiwara. A more general result is proved in [LM] but we give a simpler approach to the special case we need.

2.1 Proposition. The characteristic variety of $\mathcal{O}_{V}\left[f^{-1}\right]$ is

$$
\left\{(v, \theta) \in \mathrm{T}^{\star} V: \theta \wedge d f(v)=0 \quad \text { and } \quad f(v) \theta=0\right\} .
$$

In particular, it contains $\mathrm{T}_{0}^{\star} V$, the fibre at the origin of $\mathrm{T}^{\star} V$.

Proof. Define

$$
W^{\prime}=\left\{(v, \theta) \in \mathrm{T}^{\star} V: \theta=s(d f / f)(v), \quad f(v) \neq 0 \text { and } s \in \mathbb{C}^{\times}\right\},
$$

and let $W$ be the (Zariski) closure of $W^{\prime}$ in $\mathrm{T}^{\star} V$.

It is is proved in [SKKO, Appendix] after [K, Proposition 6.2] (see also Gi] Theorem 3.3], Gy, §2.4]) that the characteristic variety of $\mathcal{O}_{V}\left[f^{-1}\right]$ is equal to

$$
\{(v, \theta) \in W: f(v) \theta=0\} .
$$

Let $y_{1}, \ldots, y_{n}$ be a basis of $V$ and $x_{1}, \ldots, x_{n}$ the dual basis of $V^{\star}$. Write $S=$ $S\left(V^{\star}\right)=\mathbb{C}\left[x_{1}, \ldots, x_{n}\right]$ and write $J$ for the Jacobian ideal $\sum_{i} \partial f / \partial x_{i} S$. Let

$$
\widetilde{W}=\left\{(v, \theta) \in \mathrm{T}^{\star} V: \theta \wedge d f(v)=0\right\} .
$$

Now $\widetilde{W}=\mathcal{Z}(I)$, where $I$ is the ideal of $S\left(V^{\star} \times V\right)=S\left[y_{1}, \ldots, y_{n}\right]$ generated by

$$
y_{j} \partial f / \partial x_{i}-y_{i} \partial f / \partial x_{j}
$$

Since $\partial f / \partial x_{1}, \ldots, \partial f / \partial x_{n}$ is a regular sequence, the map $S\left[y_{1}, \ldots, y_{n}\right] / I \rightarrow S_{S}(J)$ defined by $y_{i}+I \mapsto \partial f / \partial x_{i}$ is an isomorphism. But the symmetric algebra is a domain by [Ei, Ex. 17.14]. Hence $\widetilde{W}$ is a closed irreducible subset of $\mathrm{T}^{\star} V$. 
Note that $(v, \theta) \in \widetilde{W}$ if and only if $d f(v)$ and $\theta$ are linearly dependent. As $f$ is quasihomogeneous and non-degenerate, we have $d f(v)=0$ if and only if $v=0$. Thus,

$$
\widetilde{W}=\left\{(v, \theta) \in \mathrm{T}^{\star} V: \theta=s d f(v) \text {, for some } s \in \mathbb{C} \text {, or } v=0\right\} .
$$

It follows that

$$
W^{\prime}=\widetilde{W} \cap\{(v, \theta): f(v) \theta \neq 0\}
$$

Therefore the Zariski closure of $W^{\prime}$ must be contained in $\widetilde{W}$; since the latter is irreducible, $W=\widetilde{W}$. It is now clear that $\operatorname{Ch}\left(\mathcal{O}_{V}\left[f^{-1}\right]\right)$ is as in the statement and contains $\mathrm{T}_{0}^{\star} V$.

The module that plays the crucial rôle in the proof of the theorem is not $\mathcal{O}_{V}\left[f^{-1}\right]$ itself but

$$
\mathcal{H}=\mathcal{O}_{V}\left[f^{-1}\right] / \mathcal{O}_{V}
$$

This $\mathcal{D}_{V}$-module is isomorphic to $\mathcal{H}_{[C]}^{1}\left(\mathcal{O}_{V}\right)$, the first local cohomology sheaf with support in $C$. It is a regular holonomic $\mathcal{D}_{V}$-module, by [BK Proposition 1.3(7)]. We next show that $\mathrm{Ch}(\mathcal{H})$ contains $\mathrm{T}_{0}^{\star} V$.

2.3 Proposition. $\operatorname{Ch}(\mathcal{H})=\mathrm{T}_{C}^{\star} V \cup \mathrm{T}_{0}^{\star} V$.

Proof. Let $U=\mathrm{T}^{\star} V \backslash \mathrm{T}_{0}^{\star} V$. Note that $U \cap \mathrm{Ch}(\mathcal{H})=\mathrm{Ch}\left(\left.\mathcal{H}\right|_{V^{\prime}}\right)=\mathrm{T}_{C^{\prime}}^{\star} V^{\prime}$. It follows at once that $\operatorname{Ch}(\mathcal{H}) \supseteq \mathrm{T}_{C}^{\star} V$. Further, if there exists another irreducible component of $\mathrm{Ch}(\mathcal{H})$, then it contains $\mathrm{T}_{0}^{\star} V$.

Recall that $\mathcal{H} \cong \mathcal{H}_{[C]}^{1}\left(\mathcal{O}_{V}\right)$. Now, (2.2) implies that

$$
\operatorname{Ch}\left(\mathcal{O}_{V}\left[f^{-1}\right]\right)=\operatorname{Ch}\left(\mathcal{O}_{V}\right) \cup \operatorname{Ch}(\mathcal{H}) .
$$

By Proposition 2.1, the fibre $\mathrm{T}_{0}^{\star} V$ is contained in the left-hand side, but not in the characteristic variety of $\mathcal{O}_{V}$, which is the zero section of $\mathrm{T}^{\star} V$. Hence $\mathrm{Ch}(\mathcal{H})$ contains $\mathrm{T}_{0}^{\star} V$, and the proposition is proved.

Recall that the link of $\mathcal{Z}(f)$ is the intersection of $C$ with a small sphere centred at the origin. Recall also the definition of the Bernstein polynomial [Co, p. 94]. This is the polynomial $b(s)$ in an indeterminate $s$ which has least degree amongst polynomials for which there exists a differential operator $p$ with the property that $p f^{s+1}=b(s) f^{s}$. From the definition, it is clear that $b(s)=(s+1) \tilde{b}(s)$.

2.4 Proposition. The following are equivalent:

(1) $\mathcal{H}$ is simple;

(2) the link of $\mathcal{Z}(f)$ is a $\mathbb{Q}$-homology sphere;

(3) $\tilde{b}(s)$ has no integer roots.

Proof. Let $i: C^{\prime} \rightarrow V^{\prime}$ be the inclusion. Then $\left.\mathcal{H}\right|_{V^{\prime}} \cong \mathcal{H}_{\left[C^{\prime}\right]}^{1}\left(\mathcal{O}_{V^{\prime}}\right)$ is simple since, by Kashiwara's equivalence (see [Bo, Theorem VI.7.13(ii), Theorem VI.7.4(i)(ii)(iii)]), one has that $i_{+}\left(\mathcal{O}_{C^{\prime}}\right) \cong \mathcal{H}_{\left[C^{\prime}\right]}^{1}\left(\mathcal{O}_{V^{\prime}}\right)$. It follows from this (see [BK, Proposition 8.5]) that $\mathcal{H}$ has a simple socle $\mathcal{L}$ and that $\mathcal{H} / \mathcal{L} \cong \mathcal{S}^{t}$ is a direct sum of $t$ copies of the simple module $\mathcal{S}$ supported only at the origin. Now consider the Riemann-Hilbert 
solution functor $S=R \mathcal{H} \operatorname{om}_{\mathcal{D}_{V}}\left(\ldots, \mathcal{O}_{V}\right)$ which gives a (contravariant) equivalence between the category of regular holonomic $\mathcal{D}_{V}$-modules and the category of perverse sheaves on $V\left[\mathrm{BO}\right.$, Theorem VIII.14.4]. One has $S(\mathcal{H})=\mathbb{C}_{C}[-1]$, where $\mathbb{C}_{C}$ is extended by zero to a sheaf on $V$, and likewise $S(\mathcal{S})=\mathbb{C}_{\{0\}}[-n]$. Further, by BK, Theorem 8.6], one has $S(\mathcal{L})=\mathcal{I C}_{C}[-1]$, the (shifted) intersection cohomology complex [GM]. Thus, one has a short exact sequence:

$$
0 \rightarrow \mathbb{C}_{\{0\}}[-n]^{t} \rightarrow \mathbb{C}_{C}[-1] \rightarrow \mathcal{I C}_{C}[-1] \rightarrow 0 .
$$

Since $\mathcal{K}=\mathbb{C}_{\{0\}}[-n]^{t}$ is concentrated in degree $n$, and $\mathbb{C}_{C}[-1]$ is concentrated in degree 1 , we see that in the bounded derived category $\mathcal{K} \cong \mathcal{H}^{n-2}\left(\mathcal{I C}_{C}\right)$. On the other hand, using the Whitney stratification $C=C^{\prime} \cup\{0\}$ and Deligne's approach to $\mathcal{I C}$ GM, §3], we get

$$
\mathcal{I C}_{C} \cong \tau_{\leq n-2} R j_{*} \mathbb{C}_{C^{\prime}},
$$

where $j: V^{\prime} \rightarrow V$ is the inclusion and $\tau$ is truncation. It follows that $\mathcal{K}$ is the skyscraper sheaf at the origin with stalk

$$
\lim _{\epsilon \rightarrow 0} H^{n-2}\left(C^{\prime} \cap B_{\epsilon}, \mathbb{C}\right) .
$$

This limit is $H^{n-2}(K, \mathbb{C})$, where $K$ is the link of $C$.

It remains to remark that $K$ is a $\mathbb{Q}$-homology sphere if and only if $H^{n-2}(K, \mathbb{C})=$ 0 . But $K$ is a compact oriented $(2 n-3)$-manifold which is $(n-3)$-connected, by [Mi, Theorem 5.2], and so by the Hurewicz theorem and Poincaré duality we have

$$
H_{0}(K, \mathbb{C}) \cong H_{2 n-3}(K, \mathbb{C})^{*} \cong \mathbb{C} \quad \text { and } \quad H_{n-2}(K, \mathbb{C}) \cong H_{n-1}(K, \mathbb{C})^{*},
$$

with all other homology groups equal to zero. The equivalence of (1) and (2) follows.

Finally, let $F$ denote the Milnor fibre, $F=\mathcal{Z}(f-1)$. The monodromy $F \rightarrow$ $F$, given by $v \mapsto e^{2 \pi i / r} v$, induces an operator on $H_{n-1}(F, \mathbb{C})$. Further, by Di Proposition 3.4.7], $K$ is a $\mathbb{Q}$-homology sphere if and only if 1 is not an eigenvalue for the monodromy operator. However, by [Ma, Théorème 5.4], 1 is an eigenvalue of the monodromy operator if and only if $\tilde{b}$ has an integer root.

\section{Proof of theorem 1.1}

Suppose that there exists a $\mathcal{D}_{V}$-module $\mathcal{M}$ such that $\mathrm{Ch}(\mathcal{M})=\mathrm{T}_{C}^{\star} V$ and let us aim at a contradiction. Since $\mathrm{T}_{C}^{\star} V$ is irreducible, we may as well assume that $\mathcal{M}$ is simple. Note that $C^{\prime}$ is homotopy equivalent to the link of $C$ and so $C^{\prime}$ is simply connected.

It follows from Kashiwara's equivalence ([Bo Theorem VI.7.11]) that $\left.\mathcal{M}\right|_{V^{\prime}}=$ $i_{+}(\mathcal{N})$, for some simple $\mathcal{D}_{C^{\prime}}$-module $\mathcal{N}$ with characteristic variety $\mathrm{T}_{C^{\prime}}^{\star} C^{\prime}$. Since $C^{\prime}$ is simply connected, $\mathcal{N} \cong \mathcal{O}_{C^{\prime}}$. Thus, $\left.\mathcal{M}\right|_{V^{\prime}} \cong \mathcal{H}_{\left[C^{\prime}\right]}^{1}\left(\mathcal{O}_{V^{\prime}}\right)$.

Now, looking at the characteristic variety, the support of $\mathcal{M}$ is not concentrated at the origin. Thus, if $j: V^{\prime} \rightarrow V$ is the canonical embedding, there is a monomorphism $k: \mathcal{M} \rightarrow \mathcal{G}:=j_{*}\left(\left.\mathcal{H}\right|_{V^{\prime}}\right)$. Of course, $\left.k(\mathcal{M})\right|_{V^{\prime}}=\left.\mathcal{G}\right|_{V^{\prime}}$. Likewise, $\mathcal{H}$ is a submodule of $\mathcal{G}$ with $\left.\mathcal{H}\right|_{V^{\prime}}=\left.\mathcal{G}\right|_{V^{\prime}}$. Since the quotient $\mathcal{G} / k(\mathcal{M})$ can only possibly be supported at the origin, we cannot have $\mathcal{H} \cap k(\mathcal{M})=0$ and so $\mathcal{H}=k(\mathcal{M}) \cong \mathcal{M}$.

Of course, as $\mathrm{T}_{C}^{\star} V$ is irreducible, $\mathrm{Ch}(\mathcal{M})$ doesn't contain the zero fibre $\mathrm{T}_{0}^{\star} V$. On the other hand, by Proposition 2.3, we have that $\mathrm{Ch}(\mathcal{H}) \supseteq \mathrm{T}_{0}^{\star} V$. This contradiction completes the proof of the theorem. 


\section{EXAMPLES}

It is now easy to give many examples: let $f=x_{1}^{a_{1}}+\cdots+x_{n}^{a_{n}}$ with $n \geq 4$, and each $a_{i} \geq 2$. Consider the graph $G$ with vertices $1, \ldots, n$ and an edge linking $i$ and $j$ if and only if $h c f\left(a_{i}, a_{j}\right)>1$. Let $G_{e v}$ denote the component of $G$ containing all the $i$ with $a_{i}$ even. Suppose that either (a) $G$ contains an isolated point, or (b) $G_{e v}$ contains an odd number of vertices and $h c f\left(a_{i}, a_{j}\right)=2$, for all distinct $i, j$ in $G_{e v}$. After Brieskorn $[\mathrm{Br}], f$ satisfies the hypotheses of the theorem. Note that the example in the introduction is a special case of this one.

We complete the paper by showing that some conormal varieties of quasihomogeneous non-degenerate hypersurfaces are characteristic varieties.

Example. Let $f=\sum_{i=1}^{n} x_{i}^{2}$ and suppose that $n \geq 4$. Then $T_{C}^{\star} V$ is a characteristic variety if and only if $n$ is even.

Proof. The case when $n$ is odd is covered by Theorem 1.1 and the previous example. So suppose that $n$ is even and let $p=\sum_{i=1}^{n}\left(\partial / \partial x_{i}\right)^{2}$. Then,

$$
p f^{s+1}=4(s+1)\left(s+\frac{n}{2}\right) f^{s} .
$$

It follows that if $s=-\frac{n}{2}$, then $p$ annihilates $f^{s+1}$. Thus, the symbol of $p$ vanishes on $\operatorname{Ch}(\mathcal{G})$ where $\mathcal{G}$ is the submodule $\left(\mathcal{D} f^{s+1}+\mathcal{O}\right) / \mathcal{O}$ of $\mathcal{H}$. Now, $\operatorname{Ch}(\mathcal{G}) \subseteq \operatorname{Ch}(\mathcal{H})$ and hence either $\mathrm{Ch}(\mathcal{G})=\mathrm{T}_{C}^{\star} V$ or $\mathrm{Ch}(\mathcal{G})=\mathrm{T}_{C}^{\star} V \cup \mathrm{T}_{0}^{\star} V$. Since the symbol of $p$ doesn't vanish on $\mathrm{T}_{0}^{\star} V$ we see that the second possibility does not occur.

\section{REFERENCES}

[Bo] A. Borel et al., Algebraic D-modules (J. Coates and S. Helgason, eds.), Perspectives in Mathematics, 2, Academic Press, Orlando, 1987. |MR 89g:32014

[Br] E. Brieskorn, Beispiele zur Differentialtopologie von Singularitäten, Invent. Math 2 (1966), 1-14. MR 34:6788

[BK] J.-L. Brylinski and M. Kashiwara, Kazhdan-Lusztig Conjecture and Holonomic Systems, Invent. Math. 64 (1981), 387-410. MR 83e:22020

[CL] S.C. Coutinho and D. Levcovitz, Involutive varieties with smooth support, J. Algebra 192 (1997), 183-199. MR 98f:32010

[Co] S.C. Coutinho, A primer of Algebraic D-modules, London Math. Soc. Student Texts 33, Cambridge University Press, 1995. MR 96j:32011.

[Di] A. Dimca, Singularities and topology of hypersurfaces, Springer, 1992. MR 94b:32058

[Ei] D. Eisenbud, Commutative Algebra with a View Toward Algebraic Geometry, Graduate Texts in Math. 150, Springer, 1995. MR 97a:13001

[Ge] I.M. Gelfand, M.M. Kapranov and A.V. Zelevinsky, Discriminants, resultants and multidimensional determinants, Birhäuser, Boston-Basel-Berlin, 1994. MR 95e:14045

[Gi] V. Ginsburg, Characteristic varieties and vanishing cycles, Invent. Math. 84 (1986), 327-402. MR 87j:32030

[GM] M. Goresky and R. MacPherson, Intersection homology II, Invent. Math. 72 (1983), 77-129. MR 84i:57012

[Gy] A. Gyoja, Theory of prehomogenous vector spaces without regularity condition, Publ. RIMS, Kyoto University 27 (1991), 861-922. MR 93f:22018

[K] M. Kashiwara, B-functions and holonomic systems (Rationality of roots of b-functions), Invent. Math. 38 (1976), 33-53. MR 55:3309

[LM] D.T. Lê and Z. Mebkhout, Variétés caractéristiques et variétés polaires, C.R. Acad. Sc. Paris 296 (1983), 129-132. MR 84g:32018

[Ma] B. Malgrange, Le polynôme de Bernstein d'une singularité isolée, Lect. Notes in Math. 459, Springer, 1975, pp. 98-119. [MR 54:7485

[Ma2] B. Malgrange, Motivations and introduction to the theory of $\mathcal{D}$-modules, Computer algebra and differential equations (E. Tournier, ed.), London Mathematical Society 
Lecture Note Series, 193, Cambridge University Press, Cambridge, 1994, pp. 3-20. MR 95h:32008

[Mi] J. Milnor, Singular points of complex hypersurfaces, Annals of Mathematical Studies, 61, Princeton University Press, Princeton, 1968. MR 39:969

[SKKO] M. Sato, M. Kashiwara, T. Kimura and T. Oshima, Micro-local analysis of prehomogeneous vector spaces, Invent. Math. 62 (1980), 117-179. MR 83g:32016

Departamento de Ciências da Computação, Universidade Federal do Rio de Janeiro, P.O. Box 68530, 21945-970 Rio de Janeiro, RJ, BraziL

E-mail address: collier@impa.br

Pure Mathematics Section, School of Mathematics and Statistics, University of Sheffield, Hicks Building, Sheffield S3 7RH, United Kingdom

E-mail address: m.holland@shef.ac.uk

URL: http://www.shef.ac.uk/ ${ }^{\mathrm{ms} / \mathrm{staff} / \mathrm{holland}}$

Instituto de Ciências Matemáticas e Computação, Universidade de São Paulo, P.O. Box 668, 13560-970 SÃo CARlos, SP, BrazIL

E-mail address: lev@icmsc.sc.usp.br 